\title{
Testing of the Prototype Receiver for ITER ECE Diagnostic
}

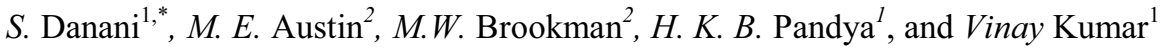 \\ ${ }^{1}$ ITER-India, Institute for Plasma Research, Bhat, Gandhinagar-382428, India \\ ${ }^{2}$ University of Texas at Austin, Austin, TX, USA
}

\begin{abstract}
The Electron Cyclotron Emission (ECE) diagnostic system in ITER provides essential information for plasma control and for evaluating the plasma performance. It measures the electron temperature profile (edge/core), electron temperature fluctuations and radiated power in the electron cyclotron frequency range from the plasma. Virginia Diodes Inc. (VDI) has designed and developed a stateof-the-art prototype receiver to detect emission in the 200-300 GHz frequency range. The prototype receiver developed by VDI has been tested at DIII-D tokamak to check its performance by measuring higher frequency ECE harmonics in high temperature plasmas. Bench testing verifies the receiver's band coverage and noise temperatures. Good sensitivity has been obtained meeting the ITER requirement. Also, the receiver performance is assessed for ITER by comparing the data from the receiver and the existing Michelson interferometer for the same DIII-D plasma shot. The measurement results show that the receiver provides accurate temperature information for the plasma emission throughout the frequency band.
\end{abstract}

\section{Introduction}

For ITER high toroidal field operation $\left(\mathrm{B}_{\mathrm{t} 0} \sim 5.3 \mathrm{~T}\right)$, the first and second harmonic ECE extends from 122-230 $\mathrm{GHz}$, and $220-340 \mathrm{GHz}$ respectively. Thus the ITER ECE receivers need to be extremely broadband $(\sim 100$ $\mathrm{GHz}$ ) for instantaneous frequency coverage and should have very good sensitivity, linearity across the very wide band, and very high reliability [1]. Similar measurement instruments have been successfully deployed in some tokamaks, like the high frequency radiometer installed in Alcator-C which operates from 234-306 GHz [2]. But the ITER system presents significant new challenges in terms of reliability and robustness. To achieve these goals, Virginia Diodes Inc. (VDI) has designed and developed prototype receiver based on advanced solid state technology [3]. The prototype receiver developed by VDI for frequency range $200-300 \mathrm{GHz}$ was installed at DIII-D to check its performance by carrying out some bench-top measurements, and then measurement of ECE radiation temperature of the DIII-D plasma to test and verify other important parameters [4]. The DIII-D ECE frequency range for third to fifth harmonics that extends from 120-300 $\mathrm{GHz}$ and the typical plasma electron temperatures of order $5 \mathrm{KeV}$, makes it suitable for testing of ITER ECE prototype receiver. In this paper, the test results of noise temperature and sensitivity of the prototype receiver are presented. The receiver performance is assessed for ITER requirement by comparison of ECE measurements with those from the DIII-D Michelson interferometer.

\section{Prototype Receiver}

The prototype receiver from VDI (Rx149), is a compact solid state millimeter wave receiver system which employs a waveguide-based triplexer, a Dielectric Resonator Oscillator (DRO) based local oscillator, an integrated tripler, subharmonic mixer and amplifier. This integrated assembly leads to improved performance and reliability. The waveguide based triplexer divides the frequency range $200-300 \mathrm{GHz}$ into three bands, low, mid, and high, which then go to respective mixer modules as shown in Fig. 1. These mixers are driven by DROs whose frequencies are set such that each frequency band is down-converted to identical IF band 5-38.33 GHz.

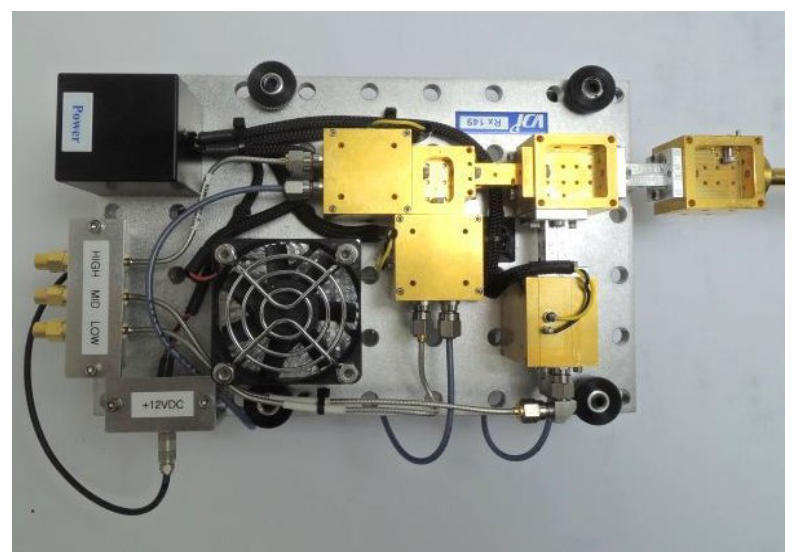

Fig. 1. VDI Prototype Receiver

This satisfies another important requirement for ITER radiometer to have identical IF section in order to 
have a simple and cost-effective system. The frequency range corresponding to the low, mid and high bands is 200-233.33 GHz, 233.33-266.66 GHz, and 266.66-300 $\mathrm{GHz}$ respectively.

\section{Bench Testing of the Receiver}

The receiver has been first tested with the VDI AMC 446 source. This is an amplifier-multiplier chain with input frequency 11-17 GHz, multiplication factor of 18 , output frequency range $200-300 \mathrm{GHz}$ and output power of $400 \mu \mathrm{W}$. This measurement is carried out to test receiver's band coverage for each of the three IF bands (high, mid, and low) and is shown in Fig. 2.

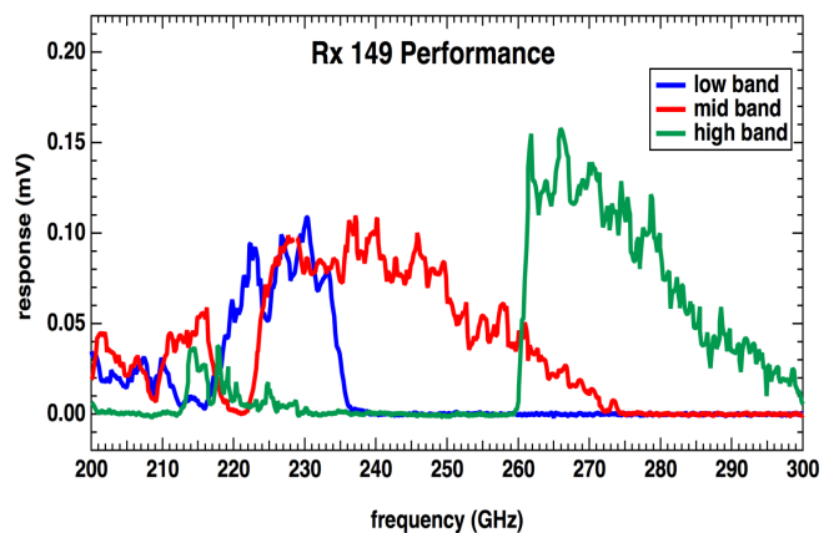

Fig. 2. Receiver performance for the three bands

\subsection{Noise Temperature Measurement}

An experiment was carried out to measure the receiver noise temperature. A W-band noise tube (CLARE TN165) was connected to the receiver input via a WR-10 to WR-3.5 waveguide transition. This noise tube radiates at $8000 \mathrm{~K}$ in the W-band. First, the noise tube spectrum was obtained using Michelson interferometer and then the noise tube radiation temperature in frequency range 50 to $400 \mathrm{GHz}$ was obtained by comparing the noise tube spectrum with the LN blackbody source spectrum as shown in Fig. 3.

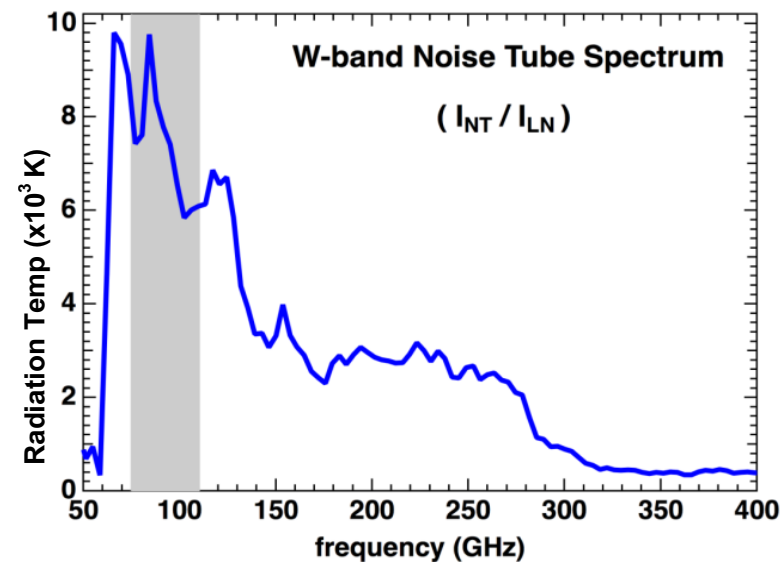

Fig. 3. Noise Tube Spectrum
Measurement indicates that the radiation temperature of the tube is at $3000 \mathrm{~K}$ for the desired receiver frequency range.

The receiver noise temperature was obtained using the Y-factor method [5],

$$
T_{r}=\left(T_{h}-Y T_{c}\right) /(Y-1)
$$

with $\mathrm{T}_{\mathrm{h}}=3000 \mathrm{~K}$ and $\mathrm{T}_{\mathrm{c}}=300 \mathrm{~K}$. The measurement of noise temperature for the three IF bands for a set of five cavity filters with centre frequencies between $2-18 \mathrm{GHz}$ is shown in Fig. 4. The values obtained are in reasonable agreement with the theoretically estimated value from VDI of $\sim 12000 \mathrm{~K}$, for mixer noise temperature of $\sim 2900$ $\mathrm{K}$.

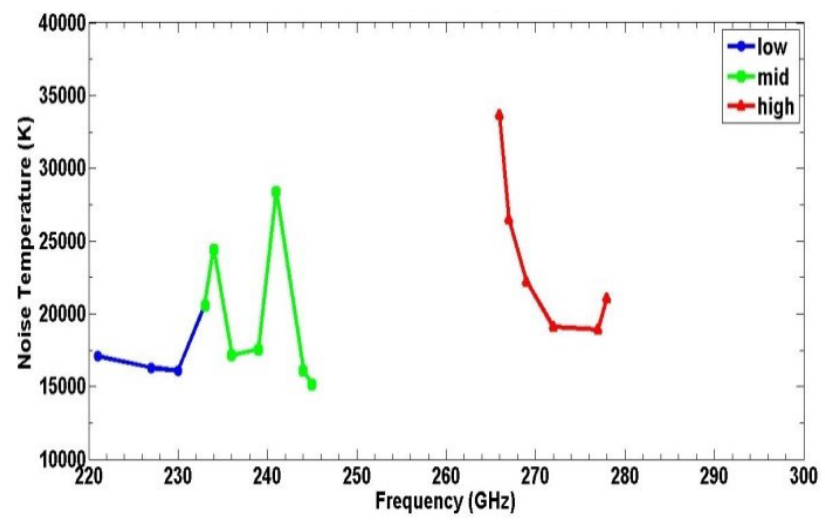

Fig. 4. Receiver Noise Temperature

\subsection{Sensitivity measurement}

VDI AMC 446 source was also used for the sensitivity measurement. First, output power from this source was measured for the full frequency band (200-300 GHz) using a power meter so that the input power at the receiver is known. This is shown in Fig. 5. For sensitivity measurement the output power was measured for the same set of filters for the three bands. Using the data of Fig. 5, an average sensitivity is obtained of $\sim 500$ $\mathrm{mV} / \mathrm{mW}$ and is shown in Fig. 6.

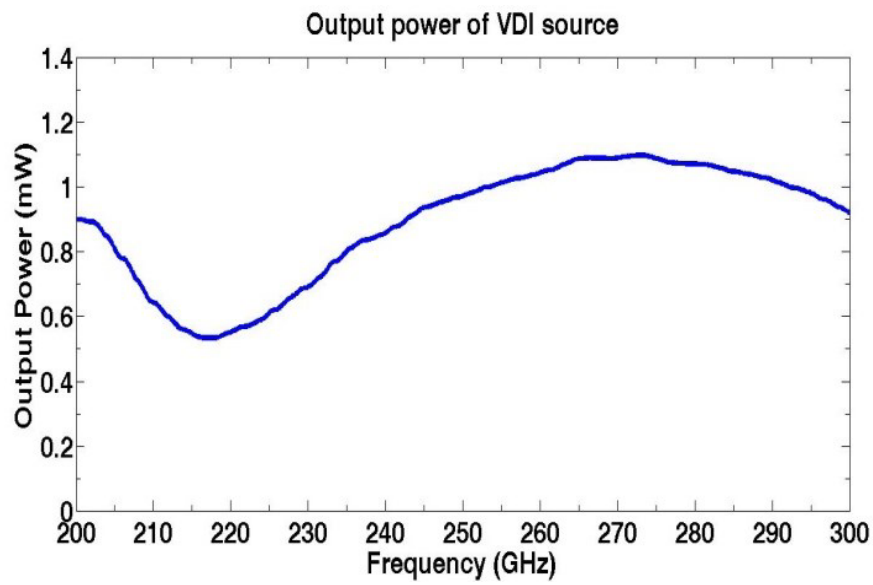

Fig. 5. Output power of VDI AMC 446 source 


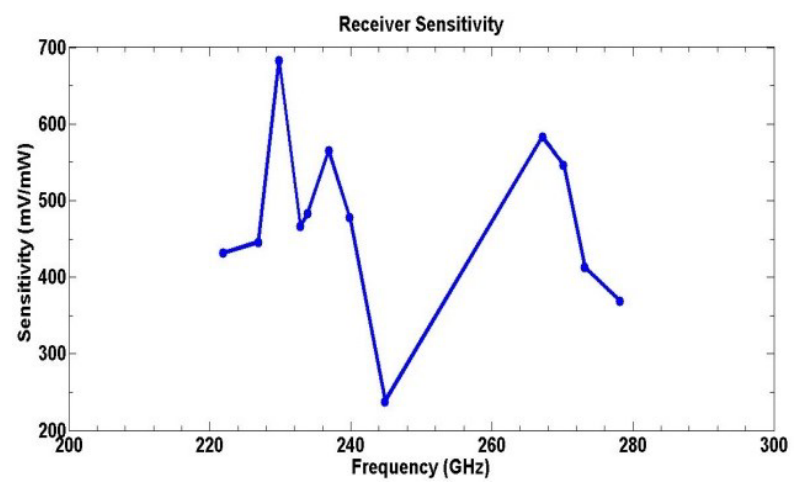

Fig. 6. Plot of receiver sensitivity

\section{Receiver Testing On DIII-D}

The prototype receiver was installed at DIII-D in a shared optical path with the Michelson interferometer [4] to facilitate simultaneous ECE measurement from both the instruments. The wire grid beam splitter positioned at $45^{\circ}$ to the horizontal divides the beam into two: one is coupled to the Michelson and, other to the receiver as shown in Fig. 7.

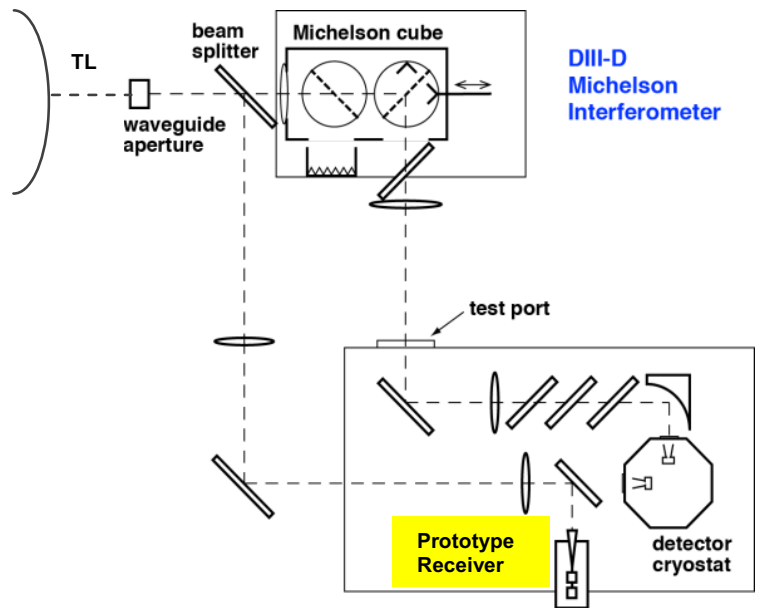

Fig. 7. Schematic of receiver set-up with Michelson at DIII-D

Fig. 8 shows the prototype receiver installed at DIII-D on the Michelson optics table.

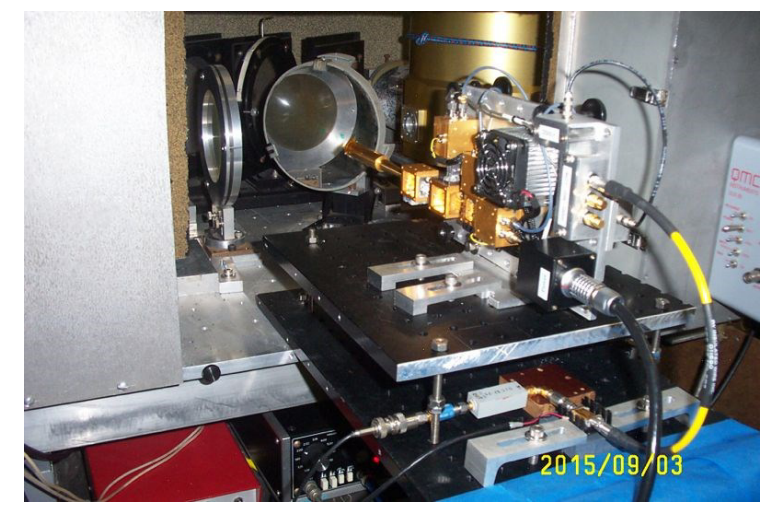

Fig. 8. Prototype receiver installed on DIII-D
A novel high intensity (3000 lumens) LED lamp source was used for the alignment of coupling optics of the receiver. The source along with an aperture is placed at the waveguide end. The image is mapped through the coupling optics into a well-defined beam spot at the opening of the receiver input horn.

\subsection{Plasma ECE Measurement}

Fig.9 shows $\mathrm{T}_{\text {rad }}$ from prototype receiver and Michelson interferometer for DIII-D shot \# 166171. It is observed that both measurements are well matched indicating that the receiver is measuring the proper frequency band. Also its response is linear.

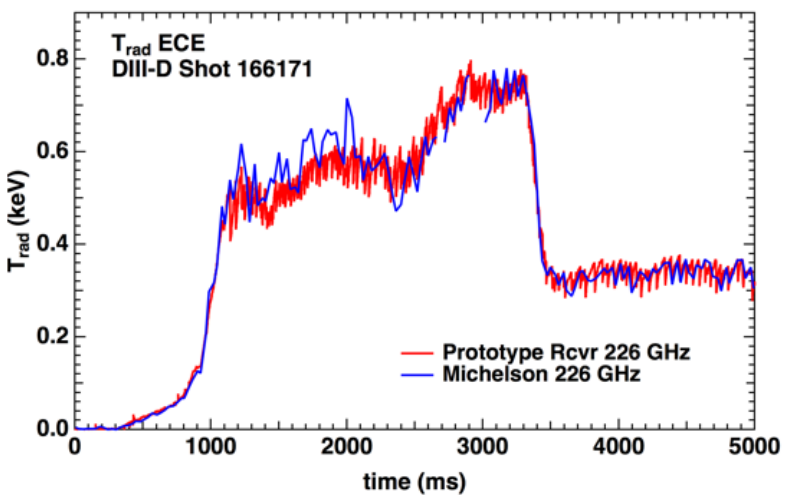

Fig. 9. Comparison of $\mathrm{T}_{\mathrm{rad}}$ from prototype receiver and Michelson interferometer for DIII-D shot 166171

\section{Summary}

Prototype receiver for ITER ECE developed by VDI has been installed at DIII-D for high harmonic ECE measurements. Bench testing of prototype receiver show reasonable values for noise temperature, sensitivity, and frequency band coverage. Measurements of DIII-D high harmonic ECE show that the receiver has linear response. Test results shows that the receiver meets ITER requirements in terms of noise temperature, sensitivity, linearity, and frequency coverage. The steady performance of the receiver even after continuous use for a long period proves its robustness.

\section{References}

1. G. Taylor et al, PPPL-5026, May 2014

2. R. Chatterjee, P.E. Phillips, J. Heard, C. Watts, R. Gandy, A. Hubbard, Fusion Eng Des 53 113(2001)

3. E.W. Bryerton, D. Koller, J. L. Hesler, and T.W. Crowe, 39th Int. Conf. on Infrared, Millimeter, and THz Waves, Tucson, AZ, September 12-19, (2014)

4. M. E. Austin, M.W. Brookman, P.E. Phillips, W.L. Rowan, S. Danani, Poster Presented at APS/DPP, Savannah, GA (2015)

5. H. J. Hartfuss and Thomas Geist, "Fusion Plasma Diagnostics with mm waves: An Introduction" Wiley Publications (2015) 